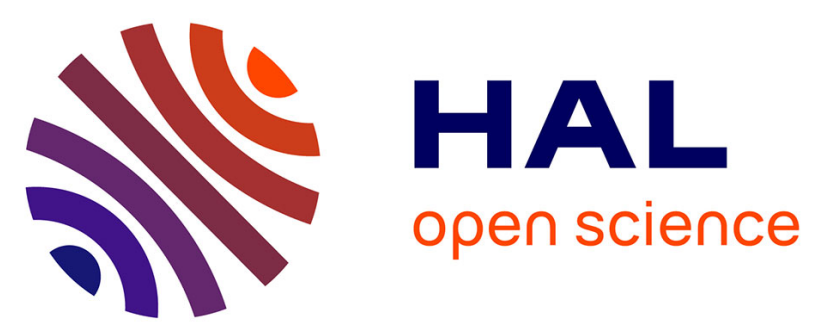

\title{
A method for Constructing a Reference Image Database to Assist with Design Process. application to the Wooden Architecture Domain.
}

\author{
Sabrina Kacher, Gilles Halin, Jean-Claude Bignon, Pascal Humbert
}

\section{- To cite this version:}

Sabrina Kacher, Gilles Halin, Jean-Claude Bignon, Pascal Humbert. A method for Constructing a Reference Image Database to Assist with Design Process. application to the Wooden Architecture Domain.. IJAC, International Journal of Architectural Computing, 2005, 3 (2), pp.227-243. 10.1260/1478077054214415 . halshs-00262180

\section{HAL Id: halshs-00262180 \\ https://shs.hal.science/halshs-00262180}

Submitted on 31 Mar 2008

HAL is a multi-disciplinary open access archive for the deposit and dissemination of scientific research documents, whether they are published or not. The documents may come from teaching and research institutions in France or abroad, or from public or private research centers.
L'archive ouverte pluridisciplinaire HAL, est destinée au dépôt et à la diffusion de documents scientifiques de niveau recherche, publiés ou non, émanant des établissements d'enseignement et de recherche français ou étrangers, des laboratoires publics ou privés. 


\section{A method for}

Constructing a Reference Image Database to Assist with Design Process. Application to the Wooden Architecture

\section{Domain}

Sabrina Kacher, Gilles Halin, Jean-Claude Bignon and Pascal Humbert 


\section{A method for Constructing a Reference Image Database to Assist with Design Process. \\ Application to the Wooden Architecture Domain}

Sabrina Kacher, Gilles Halin, Jean-Claude Bignon and Pascal Humbert

Designing architectural projects requires the introduction of references, because design is an activity oriented towards a result which does not yet exist. If we summarise the current categories used in Artificial Intelligence to characterise the different forms of reasoning, we are able to consider that design is more the concern of the induction or the abduction mechanism than the deduction mechanism. Moreover, the main characteristic of the designer's activity is to work towards non-routine situations with the use of many references. In this paper we will present method principles to construct a reference image database. These references will enable the designer to further in solving the design problem. To illustrate these reference usage, we choose photographic images belonging to the wooden construction domain We also present at the end of the paper an experiment which aims to evaluate the real help that this reference image database can bring to designers during their creation task. 


\section{Introduction}

Design requires research into ideas [I] and documentation to help designers in their creation work. It is a domain where the use of pictures (drawings, photograph, etc) is essential because the information transmitted by images is often easier to understand than the one transmitted by texts. The fact is that an image requires less interpretation than a text. The information transmitted by image (element shape, colour, light, etc) is already "put in shape" and can be more easily integrated into the design process. For this we have chosen a specific kind of image to illustrate the references for the designer which is "photographic" images.

Photographic image is widely used as an information support in the design field because it can convey a large amount of information that will help the designer to solve his design problem in a minimum of time. It also illustrates several elements in different ways and the designer tends to put at the same level of cognition the object and the image of the object.

The particularity of the design process is that it brings together two distinct operations where image use is essential. The first one concerns problem formulation (image allows the user to express his design question and enables him to advance in his problem formulation). The second one concerns the problem's solution (image illustrates several potential solutions to the design problem) [2]. These operations can be done thanks to mental images that the designer creates in his mind.

This paper tackles the particular question of the principles to follow in order to better construct a reference image database that will help the designer during the design process. We will also present a concrete application of the proposed method to a particular domain which is the wood construction domain. Finally we will present a concrete example of how our method once implemented in a suitable search engine can help designers during their design process.

\section{The method principles}

Our work consists of presenting a method to better describe, index and access images. This method is oriented toward a semantic description of images. Our proposition is not to suggest help to the designers who want to index their own image database. These are indexation principles that will be used in an information resource centre to offer an information service to designers. To construct the database we suggest following these principles:

- Defining the context of the work.

- Defining the structure of the chosen domain.

- Defining a way to quantify the visual importance of an element.

- Identifying a specific research process. 


\section{I. Defining the context of the work}

Even if we have decided to focus on the architecture domain, the person that will define and propose a semantic indexation method has to reduce and limit the field to be described. It's a necessary task because architecture is a wide-ranging domain and the objective is to define a vocabulary which aims to represent and describe as much as possible a precise domain [3]. Then this definition of the context will direct all the work to follow. Moreover all domains possess various sub-domains. For example in architecture we have to define description, construction, design, social and urban ways amongst others. To succeed with this method we have to define as much as possible the domain where we work.

\subsection{Defining the structure of the chosen domain}

Nowadays there are several fields of research being undertaken which aim at describing a domain in words. There are a lot of vocabularies applied to describe various domains (ontologies, thesauruses, lexicon, etc). The most widespread kind of existing vocabularies are "thesauruses" [4]. These thesauruses possess deep structure or hierarchy. The extents of these hierarchies meant that it took a very long time to find the suitable word to describe an element and then induce a wrong description of the document. In our case we used a proposition made by Eleanor Rosch [5] to classify the natural categories in the cognitive psychology domain. She suggested classifying all the objects of the real world on only three hierarchical levels. She explained that the categorisation could be done well starting from the intermediate level. This level includes the name of the prototype of all the current objects of the domain to be categorised. This level can include in the field of furniture, "chair", "table", etc., or in the fruit domain, "apple", "orange", etc. To construct the two other levels we have to respect some rules. The process of generalisation can be carried out by identifying the attributes of the objects named in the previous level. For example to create the superior level of "apple" and "orange" we can create the "fruit category". This category includes the shared properties of an "orange" and an "apple". To create the inferior level Rosch proposes to add a specificity to the term of the current level. For example, an "office chair" could be different from a "kitchen chair".

\subsection{Defining a way to quantify the visual importance of an element}

To describe an image in a better way we have to organise the terms inside the image indexations. Then we focus only on the description of what is visually expressible by our thesaurus words in images. In our case the visual parts concern the physical parts of architectural realisations. We propose a classification or an organisation of the terms used for image indexation. This 
classification depends on the visual significance of the illustration and is related to the angle from which the picture is shot. In some images an architectural built work should be easier to identify than in others. This happens specifically for junction elements which require a close-up shot to be recognise.

\subsection{Identifying a specific research process}

To make the problem formulation and solution easier for the designer we have defined a search mode adapted to the designer's state. Nowadays, the common access to images proposed by existing system, is the search mode using text. This mode requires using text to express a need. But in the architectural design process the needs are rarely precise and expressible in words. For this we proposed a particular kind of research mode which is the search with images. This kind of research uses images to access other images. We feel that this search mode is more adapted to the designer need during design activity. In our case the designer will use only images to formulate his need. Our system mode doesn't require text to formulate a need. We consider this search mode more adapted to the state of need which is imprecise and fuzzy especially in the earlier phases of the design process (figure 5).

\section{Application to a specific domain}

To validate the relevance of the proposed method we applied the enumerated principles to a concrete example. For this we chose a particular sub-domain of architecture, which is architecture built in wood. Then, photographic images that we work with illustrate buildings or parts of these buildings belonging to the wood construction domain.

According to the principles enumerated above, we will propose an indexation process in four steps as follows ;

\section{I. Step (I): defining the context of the work}

At the beginning, we defined a vocabulary for image indexing. Defining a vocabulary results from the analysis of images to index.

There are two main families of image describers:

- The "Offness" family which is related to the directly extractive information from the image. Its divided in two parts:

- The graphic content outline, texture, shape, etc. [7]

- The semantic content relative to the elements represented directly on the image.

- The "Aboutness" family which is related to describe the complementary information but not directly extractive information 
from images. Its divided in two parts:

- The analysis content relative to the name of the project, the type of the project, the geographical situation, etc.

- The symbolic content that is located on a more elevated abstraction level such as opacity, permeability, monotony, etc.

For this application example, we decided to focus on the part concerning the information related to the elements represented in the picture and the one related to the project.

In order to meet efficiently the user's expectations and to reduce the ambiguity resulting from the multi-interpretation of the image (polysemy), we defined four families (Figure I) of image describers [8]:

- The architectural realisation family; includes the name of the category to which the architectural element illustrated belongs (school, singlefamily dwelling, ...).

- The built-works family; indicates every physical part of an architectural realisation which has a particular function (post, beam, window, ....).

- The material family; includes every wood material and its by-products (species, glued-laminated, ...).

- The products family; includes any component aimed at protecting and decorating wooden elements (fungicide, impregnation, ...).

\section{Products}

Salts for autoclave treatment

\section{Material \\ Fir tree}

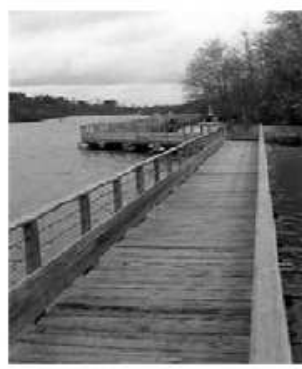

\section{Architectural realisation}

Jetty

\section{Built works}

Gard-rail half stud

Decking
Figure I. Image families of describers

\subsection{Step (2): defining the structure of the chosen domain}

The vocabulary created has to enable the formalisation of the more or less homogeneous language-practices of the professionals in the domain [9]. This 
point is important because these professionals have the same scientific and technical references. To better represent the described domain, we structured each family of image describers, following the E.Rosch [5] categorisation which structures in 3 hierarchical levels real world objects. The categorisation is carried out from the intermediate level called "current level". The definition of each level is made following rules (Figure 2) of generalisation and specialisation [I0]. The three levels are as follows [ $\mathrm{II}$ :

The current level (CL)

It represents the level from where the language is structured. This level is also called a basic level because it contains the most important concepts in the language of the domain we want to describe and therefore, the one we learn in the first place. This level corresponds to the designer's problem when the problem is of a general nature. Its contains the name of "prototype" or of the most representative member of the elements that have properties in common. It includes the name of concrete elements illustrated in the images e.g. post, beam, window, door, etc.

The superior level (SL)

It corresponds to the fuzzy demand of the user and guides him during a search towards a more precise and more specific level. This level represents a more general level than the current level. Terms belonging to this level allow identifying the shared attributes of the architectural concepts belonging to the current level. For example, a "post" is a "vertical structure element", and a "wall" is also a "vertical structure element". The shared attributes for both these elements are "structure" and "vertical". So the superior category will include these attributes and will be called "vertical structure system" category.

The inferior level (IL)

The terms belonging to this level allow a more concrete identification of the architectural concepts illustrated in the images than those of the previous levels. The concretisation corresponds to the user's demand during his search for precise architectural solutions to his design problems. Terms belonging to this level are used for indexing images and contain only one degree of extra specificity, compared to those of the current level. This specificity is obtained by the research of characteristics such as:

- The shape;

Corresponds to the "outline" of an object. This characteristic has two levels. i) the shape of the architectural element itself e.g. Post $\rightarrow$ Circular post and ii) the unit used e.g. indoor flooring $\rightarrow$ panels. 
- The constructive mechanism;

Corresponds to the way the architectural element is arranged, according to predetermined rules of construction. E.g.Wall $\rightarrow$ Halftimbered wall

- The functional mechanism;

Corresponds to the way that the architectural element is used to fulfil its characteristic role. E.g.: Cladding $\rightarrow$ Shingle cladding

- The number;

Corresponds to the multiplicity or to the uniqueness of a single architectural element. E.g. Structure $\rightarrow$ Successive porticos

- The orientation;

Corresponds to the direction of the element with regard to the horizontal / vertical or intermediate axes. E.g. Sun-breaker $\rightarrow$ Horizontal sun-breaker.

- The full-empty device;

Corresponds to the extent to which the architectural element has been opened up with the aim of letting in daylight or not. E.g.

Partition $\rightarrow$ Honeycomb wall.

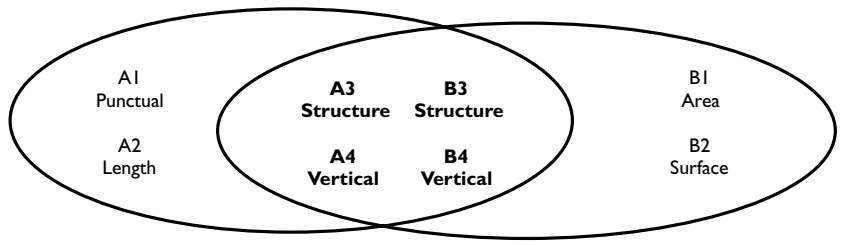

Figure 2. The vocabulary construction.

Post

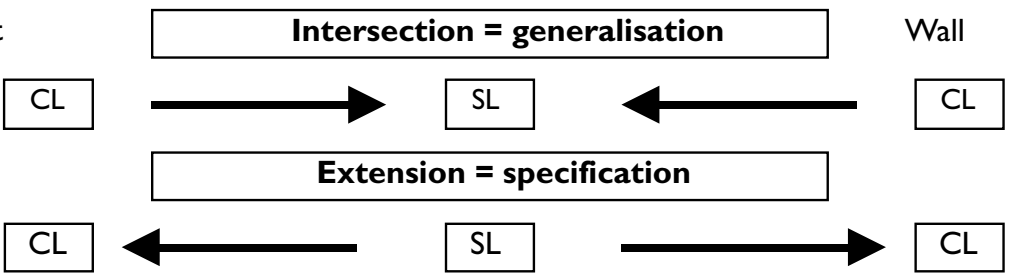

3.3. Step (3): defining a way to quantify the visual importance of an element

Describing a built-work element illustrated by an image with a vocabulary term is not enough. An element can be visually more important in one photographic image than in another one. This variability of importance is related to the shape of the element and also depends on the angle from which the view is shot (Figure 3). We organised terms used to index each image according to its visual importance. This importance must be quantified 
to be controlled. We therefore identified visual properties that will enable us to evaluate and quantify this significance [12].

Figure 3.The organisation of the indexation terms
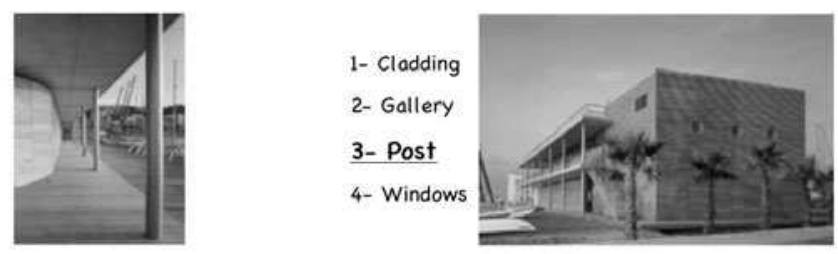

We defined five image properties (Figure 4) [6]:

The occupied area

Depends on whether the illustration of the real architectural element occupies a large area in the image or not. Thus, the representation of the element is in a prominent position if it occupies a larger visual area than the other elements which surround it.

The archetype likeness

Depends on the likeness of the illustration of the real architectural element to the ideal model shared by professionals belonging to the same domain. If the representation of the element keeps the structural and spatial properties of the real objects so allowing the viewer to identify the element appearing in the image, the element will be easier to recognise [13].

The contrast with the background

Depends on the capacity of the illustration of the real element to emerge from the rest of the image. The element will be in a conspicuous position if its representation contrasts strongly with the rest of the image (colour, light, ...).

The focus

Depends on the position of the illustration of the real architectural element in the image. If it occupies the centre of the image (diagonal junction), the element should be more obvious than the rest of the elements illustrated.

The completeness

Every illustration of a real element shows only a part of it. This graphicproperty depends on whether the illustration of the object represents the semantic features or not [14]. This allows a viewer to identify the represented element. 


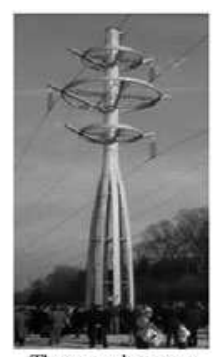

The completeness

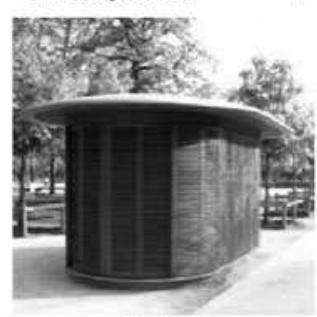

The focus

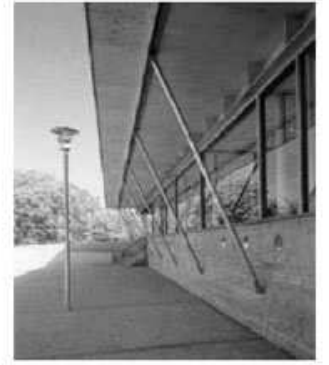

The archetype likeness

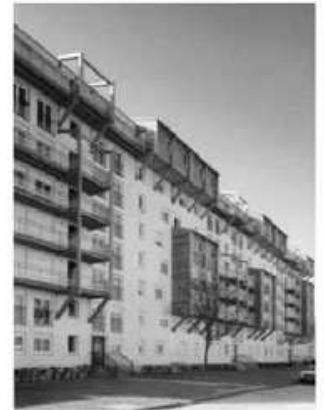

The contrast

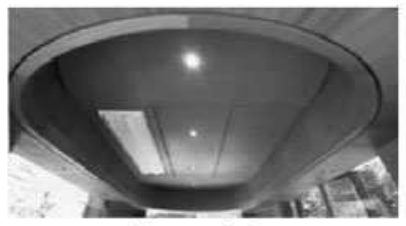

The occupied area

All these graphic properties do not possess the same impact. Their impact depends on the kind of architectural element which is illustrated in images. Thus, we group all the architectural elements named in the thesaurus according to the Ching [15] classification (planar elements, linear elements and punctual elements). This classification is oriented toward a classification of architectural elements according to their geometric features. To evaluate the real importance of each graphic property related to the geometric type of the element illustrated we carried out an experiment. This experiment has allowed us to propose calculation rules that will facilitate the terms classification task. The objective of this part is to enable the system to propose semi automatically a value that will be associated to a rank for every term used for the indexation [II, I2]. This is carried out according to the kind of built work to which the element indexed belongs. The experiment allows us to extract some observed values from the Chisquare [16] table:

- Planar elements: for planar elements the "occupied area" graphic property represents more than $72 \%$ of the chi-square value. It corresponds to the estimation of the importance that the property area has for the planar element.

- Linear elements: for example, the image in illustrates both "post" and "cladding". These architectural elements were grouped in two different categories according to their geometric properties. A post belongs to linear elements and cladding belongs to surface elements. To follow the differences between the importance of these properties we decided to give a more important value to the most important graphic property. 
The goal of this part is to define an algorithm which enables the system to propose semi automatically a weighted value for every term used to index images. Our proposition consists of an association of a double value to the first most important graphic property for each family. The value obtained with the application of these mathematical rules will constitute the weighted value of the term. This weighted value will be considered as a basis to classify the indexation terms. Consequently, this part allows us to propose steps to facilitate the indexer's task when he associates a rank with every term used for the semantic indexation of images. The steps are as follows:

I. Firstly, the indexer must identify the element represented by the image that will be described.

2. Secondly, for each selected element, its representation will be compared to the experiment results. This will enable the system to propose rates to classify the indexed terms.

3. Thirdly, the indexer will decide if she/he will validate or refuse the ranks proposed by the system during the indexation process.

\subsection{Step (4): identifying a specific research process}

The research process we used is that of the interactive and progressive research system [6] by image which has been developed by the MAP-CRAI! The defined vocabulary will be inserted into the system developed by the MAP-CRAI in order to better meet the user's needs. A method of relevance feedback is used by the system to propose new images for the user's queries. The indexing document is represented by a weighted vector of thesaurus terms according to the term classification of the image indexation. A vectorial matching model is then used between the query and the indexing document. The results of this matching will be given as an ordered list of images representing the user's choices.

In the previous section, we proposed a particular way to access images [17]. This access mode is based on a visual formulation of the designer's need. The user will only manipulate images during his research. Images will be presented in a mosaic layout, and the user will select, reject, or not give an opinion on every image that he visualises. The selection is obviously oriented by the design problem. For example if he searches for windows, all the images which illustrate window will be selected by the user, and the others will be rejected or without an opinion. The objective of this kind of selection enables the user to concentrate only on his design task. Therefore, when the designer expresses a need by text, it means that he expresses a mental image in words. In this case, the user translates graphic information into textual information. He uses part of his "mental energy" on another

T"MAP-CRAl" Architecture and landscape Modeling - Research Center in Architecture and Engineering, Architecture School of Nancy. 
task, which is "translation". Also, several research works have shown that mental images are easier to produce and to modify starting from images. For this reason we avoided using another kind of expression for the designer (Figure 5).

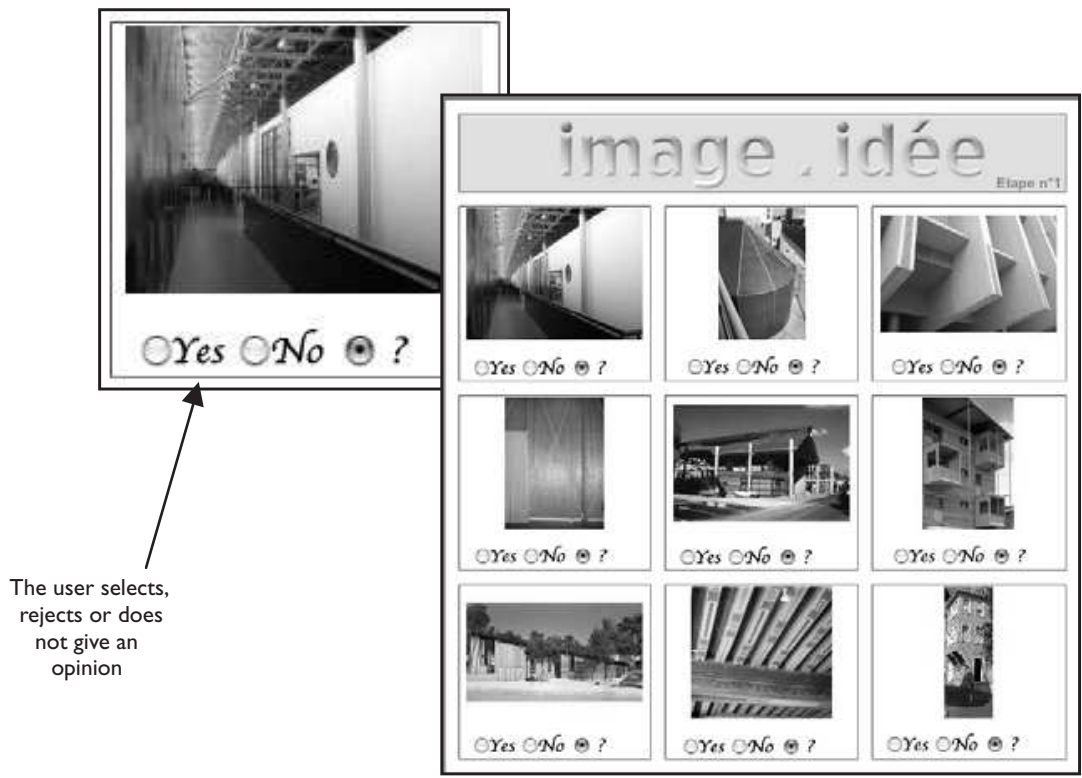

\section{Employing our method}

Once all these steps have been completed, indexers can use our method to construct an image database, and designers can use the research system to find image references.

\section{I.The indexer usage}

The indexer has to:

- Select an image to index.

- Visualise the image and identify the elements to describe.

- Use the built thesaurus and select only from the inferior level-terms which are the most suitable for describing the element identified in the image.

- Associate the selected terms with the image.

- Analyse the classification proposed by the system. For each selected element, its representation will be compared to the results obtained by this experiment. According to the results obtained though this experiment, the system proposes rates to weight the indexed terms.

- Validate or refuse the rates proposed by the system during the indexation process.

- Register the indexation operation.
Figure 5. The interface adapted to image research 


\subsection{The designer usage}

When the designer has a design problem he will use the system proposed. At the beginning he has to identify his design problem. He is not obliged to define it in words. The designer can draw it or express it another way. The efficiency of our method is based on a cognitive exchange process between the mental images of the designer and the real photographic images retrieved by the system. This happens in three steps.

- The first stage: this step concerns the state just before visualisation of the images. In this part, the designer possesses more or less vague notions and creates mental images according to his advancing state in the problem solution process. The images which come to mind are fuzzy, so he queries the system.

- The second stage: at the beginning of the research, the system presents a mosaic of images, which were selected randomly or thematically. The user selects or rejects the images which are presented to him, according to his judgement on their relevance. The system analyzes these choices, and presents to the user a new sample of images which corresponds better to the user's needs.

- The third stage:The last part of the cognitive process happens after the first visualisation of the images proposed by the system. In this part, the user shows this new sample of images and makes a permutation by replacing the elements composing his own mental images with those visualised in the real images. It operates a deformation and an adaptation of his mental images, and generates new images. This stage should allow the user to reformulate the problem, or to start a new problem which has not appeared before, or even to solve the design problem.

\section{The final experimentation}

To validate the relevance of our method, we carried out a final experimentation with ten designers in a design situation. We gave every subject a design problem and asked him to propose a timber structure covering a swimming pool with $25 \mathrm{C} 50$ meter size.

\section{I.The protocol}

The experimentation was carried out in four stages.

Stage $N^{\circ} I$

At this first stage we asked the designer to draw the first sketch (Figure 6). 


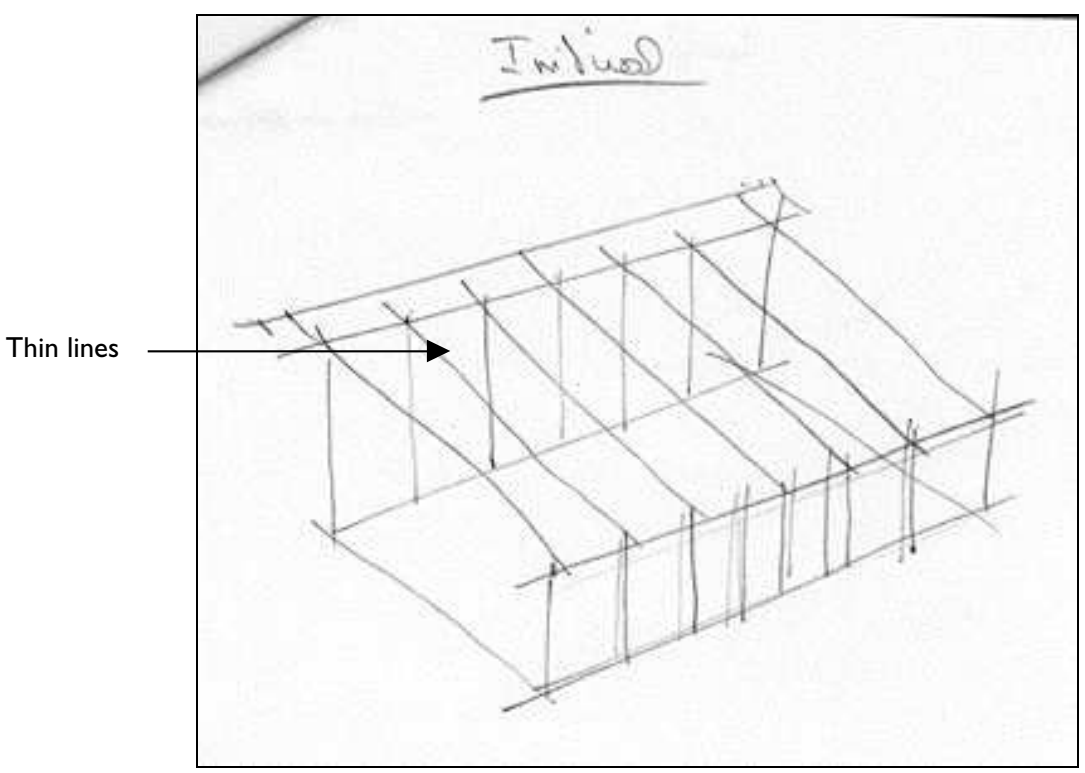
$\mathrm{N}^{\circ} \mathrm{I}$.

We can clearly identify that this first sketch is relatively purified. This drawing includes a group of similar vertical and horizontal elements. The lines representing these elements are simple. We can also notice that the shape of the roof is a sloped one and the proposed framework consists of a succession of porticos with a small roof overhang.

\section{Stage $N^{\circ} 2$}

During this stage, we asked the subject to start the research, and the first sketch was taken away from the subject.

\section{Stage $N^{\circ} 3$}

During the third stage, the subject selects, rejects or does not give any opinion on every image presented to him by the system. The last mosaic visualised is presented in the next figure (Figure $N^{\circ} 7$ ). We can see that this mosaic illustrates various built work elements such as joist, sun breaker, wind bracing, posts, etc.

\section{Step $N^{\circ} 4$}

The last stage was performed by asking the subject to redraw his early design problem (Figure 8).

\subsection{Conclusion for subject $\mathrm{N}^{\circ} \mathrm{I}$}

For this subject, we can clearly identify that the visualised images enable him to add information related to his design's problem. The final sketch looks more completed and loaded than the first sketch. The inclusion of these 
Figure 7. Last mosaic visualised by subject $\mathrm{N}^{\circ}$ I

Figure 8. Final sketch of subject $\mathrm{N}^{\circ} \mathrm{I}$.

Figure 9. The image from the mosaic which inspired the sun breaker idea.
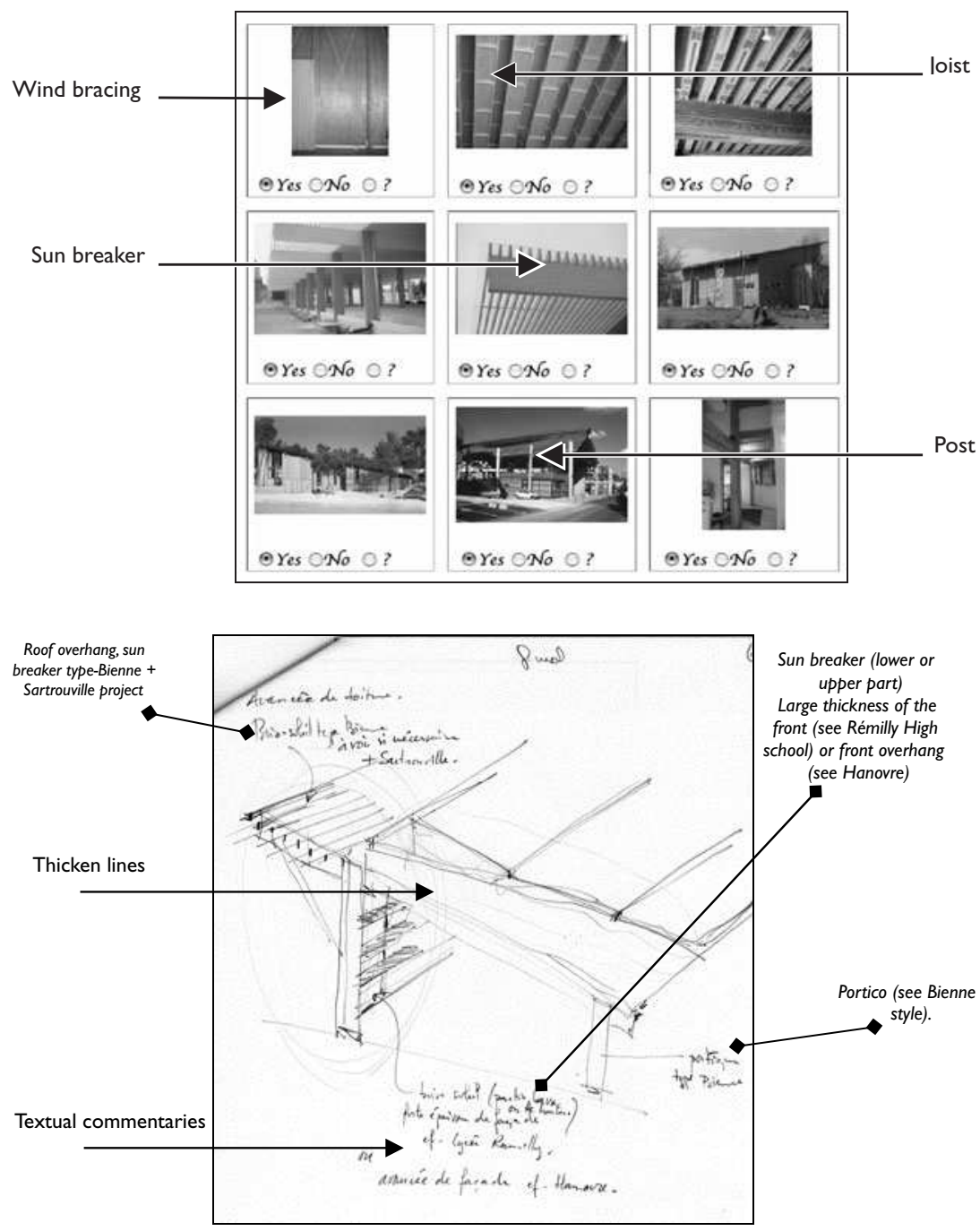

additional visualised peaces of information and the integration of this information takes place during this design exercise. We can also clearly notice that the subject added a caption or textual comments to his final sketch with some details about the material used or the junction types used. At the end of the experiment we asked the subject a series of questions. These questions were related to the identification of images which influenced his final solution for the design problem. This first subject said "at the beginning I had not idea, but this image (centre of the mosaic) gave me the sun breaker idea" (Figure 9).

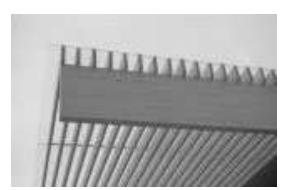


And when we asked him what the research into built work elements gave him during his design process he answered "the visualised images illustrate real architectural realisations, so we can very quickly get an idea of what the built work in question will look like once integrated into the project (we have an example of the use of this built work)".

\section{Conclusion}

In this article we present four method principles for defining a way to describe index and access images. This method aims to facilitate the construction of a photographic image database that will play the role of a reference tool for the designer during his design activity. These principles consist of defining the vocabulary adapted to the description of the domain, defining an indexation model and identifying the suitable way to access images depending on the degree of precision of the designer's need. The defined vocabulary, when associated with images, must be organised according to five visual properties. Finally, we proposed a particular way to access images without introducing text. All these principles have been applied to the specific domain of architecture built in wood.

We carried out a final experiment to evaluate if the proposed method satisfies a designer in a design situation. Thanks to the analyses of the sketches produced by the designer during the final experiment, we were able to evaluate in concrete terms the help that this kind of research can bring to designers during their creation activity.

Future works are divided in two parts. The first one concerns the application of these principles to other technical domains belonging to the architecture field. The second one concerns the use of semantic networks as another kind of navigation to access images.

\section{References}

I. Heylighen. A, IN CASE OF ARCHITECTURAL DESIGN, critique and praise of casebased design in architecture, Katholieke Universiteit Leuven Ed, may 2000.

2. Lebahar, J.-Ch.. Le dessin d'architecte, simulation graphique et réduction d'incertitude. Parenthèse Ed, Marseille, 1997.

3. Cabré,M.T, Terminology:Theory, Methods and Applications, Juan C. Sager ed, Amsterdam, Netherlands, 1999.

4. Dauzats, M, Le thésaurus de l'image: étude des langages documentaires pour l'audiovisuel, Paris, 1994.

5. Rosch, E.. Natural Categories, Cognitive Psychology, Vol. 4, issue 3, elsevier Ed 1973.

6. Bignon, J.C., Halin, G., Nakapan, W, Building Product Information Search by Images. Proceedings of the 5th International Conference in Design and Decision Support Systems in Architecture, Nijkerk, The Netherlands, Pp. 47-6I, 2000.

7. G. Duffing, Thematico-Visual Image Retrieval: How to Deal With Partially Indexed Corpora, Internet Imaging II, San Jose, California, 200I.

8. Noy Fridman, N and Mc Guinness, D.L, Ontology Development IOI:A Guide to Creating Your First Ontology, technical report, Stanford Knowledge systems 
laboratory and stanford medical Informatics, Stanford, USA, $200 \mathrm{I}$.

9. Hudon, M.. Le thesaurus, conception, elaboration, gestion., Asted ed, Ottawa, Canada, 1994

10. Aitchison, J and Gilbrichrist, A, Thesaurus construction: a practical manual, Second Edition, London, 1987.

II. Sabrina Kacher, Jean-Claude Bignon, Gilles Halin and Pascal Humbert, A semantic indexation method to assist with the architectural design process by images, Sigradi 2004, VIII Iberoamerican Congress of Digital Graphic, november 2004, Porto Alegre, Brasil.

12. Sabrina Kacher, Jean-Claude Bignon, Gilles Halin, A method to index images in the wooden architecture domain, Terms hierarchy and weight given to terms, DDSS 2004, 7th International Conference on Design \& Decision Support Systems in Architecture and Urban Planning 2 - 5 July, 2004, Netherlands

13. Denis, M. On figurative components of mental representations, In F. Klix, J. Hoffmann, \&E. van der Meer Ed, cognitive research in psychology, Amsterdam, 1982.

14. Reed, S. K. COGNITION théories et applications, De Boeck Ed, Paris, 1999.

15. Ching Francis D-K, Architecture: form, space and order, New-York:Van Nostrand Reinhold, New york, USA, 1996.

16. Joly martine, Introduction à l'anayse de l'image, Nathan Université Ed, 1993, Paris

17. Yadolah Dodge, Statistique Dictionnaire encyclopédique, Dunod Ed, 1993, Paris.

Sabrina Kacher, Gilles Halin, Jean-Claude Bignon and Pascal Humbert.

CRAI (research centre in Architecture and Engineering), Architecture school of Nancy, 2, rue Bastien Lepage - B.P. 4355400 I Nancy CEDEX, France.

kacher@crai.archi.fr,

halin@crai.archi.fr,

bignon@crai.archi.fr,

humbert@crai.archi.fr. 
